\title{
On the Crosslinking Reaction in Negative Electron Resists Based on Polystyrene and $\operatorname{Poly}(\alpha$-methylstyrene)
}

\author{
Saburo ImAmURA, Toshiaki TAmAmURA, and Osamu Kogure \\ Polymer Section, Ibaraki Electrical Communication Laboratory, \\ Nippon Telegraph and Telephone Public Corporation, \\ Tokai, Ibaraki 319-11, Japan \\ (Received January 17, 1984) \\ KEY WORDS Crosslinking Reaction / Negative Resist / Electron Resist /
Chlorinated Polystyrene / Chloromethylated Polystyrene /
Chloromethylated Poly $(\alpha$-methylstyrene) /
}

Negative resists based on polystyrene (PSt) are now considered as one of the most important materials for microfabrication involving dry-etching techniques, owing to the relatively high stability of the benzene ring against various plasma reactions. Since the sensitivity of PSt toward high energy beam exposure is so low, ${ }^{1}$ various crosslinkable units were introduced into the PSt structure for the sensitization. Halogen groups were found to be particularly suitable to obtain both high resolution and sensitivity. Polychlorostyrene (PCSt), ${ }^{2}$ chloromethylated PSt (CMS) ${ }^{3}$ and iodinated PSt (IPS) ${ }^{4}$ are representative negative electron resists developed so far for direct wafer processing.

The crosslinking reaction in this class of resists is believed to occur via radical species produced by the cleavage of carbon-halogen bonds. In halogenated PSts where no unsaturated reactive group exists, a stepwise reaction could be a main mode for crosslinking. The participation of hydrogen atoms at the $\alpha$-position of the PSt main chain in the crosslinking reaction has been suggested, because $\alpha$-hydrogen is easily subtracted to form a relatively stable benzyl radical. However, the crosslinking reaction scheme involving $\alpha$-hydrogen and the degree of its contribution to the over-all reaction remain unclarified.

Recently, a very high contrast negative electron resist chloromethylated poly $(\alpha$-methylstyrene) ( $\alpha$ M-CMS) was synthesized by our group. ${ }^{5}$ In $\alpha \mathrm{M}$-CMS, the starting polymer for chloromethylation was changed from PSt in CMS to poly ( $\alpha$-methylstyrene) (PMSt), where the $\alpha$-position is substituted by a methyl group. Despite their structural similarity, $\alpha \mathrm{M}$ CMS shows a significantly higher contrast than CMS. The $\alpha \mathrm{M}-\mathrm{CMS}$ sensitivity is about half that of CMS, but still 30 times higher than that of PSt, when the polymers of same molecular weight are used. This difference between $\alpha \mathrm{M}-\mathrm{CMS}$ and CMS suggests the effect of the hydrogen at the $\alpha$-position on the crosslinking mechanism.

In this paper, we present the direct evidence for the participation of the $\alpha$-hydrogen in the PSt main chain in the crosslinking reaction of halogenated polystyrenes.

\section{EXPERIMENTAL}

Our method was utilized a mixture of highly sensitive halogenated PSt and a starting polymer with low sensitivity. As halogenated polymers, CMS, $\alpha \mathrm{M}-\mathrm{CMS}$, and PCSt were used, and as plain polymers PSt and PMSt. Fig- 


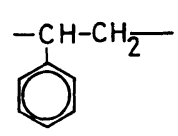

PSt

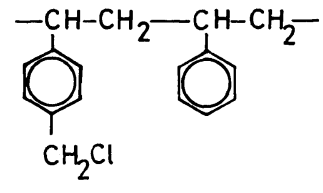

CMS<smiles>CCC(C)c1ccc(Cl)cc1</smiles>

PCSt

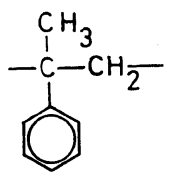

PMSt

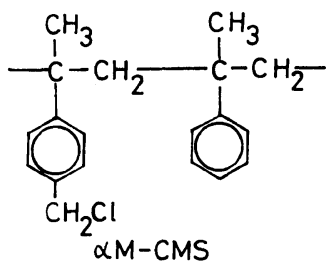

Figure 1. Chemical structure of aromatic polymers used. PSt, polystyrene; CMS, chloromethylated polystyrene; PCSt, poly(4-chlorostyrene); PMSt, poly( $\alpha$-methylstyrene); $\alpha \mathrm{M}-\mathrm{CMS}$, chloromethylated poly $(\alpha$-methylstyrene).

ure 1 shows the structures of these polymers. CMS, PCSt, and PSt have $\alpha$-hydrogens, but not $\alpha$ M-CMS and PMSt do not. CMS and $\alpha \mathrm{M}-\mathrm{CMS}$, in which the chloromethylation ratio (CR) was more than $90 \%$, were synthesized as previously reported. ${ }^{3,5}$ The PSt and PMSt used were nearly monodispersed $\left(M_{w} / M_{n}=1.05-1.1\right.$ from Toyo Soda). CMS and $\alpha \mathrm{M}-\mathrm{CMS}$ also had very narrow molecular weight distributions. PCSt was obtained by radical polymerization of 4chlorostyrene. Its $M_{w}$ and $M_{w} / M_{n}$ were $2.2 \times 10^{4}$ and 1.4 , respectively.

To obtain uniform and transparent films from polymer mixtures, various solvents including xylene, chlorobenzene, methyl isobutyl ketone ethyl cellosolve, and methyl cellosolve acetate were tried. Silicon wafers with a $\mathrm{SiO}_{2}$ surface were used as substrates, and baked at $800^{\circ} \mathrm{C}$ for $2 \mathrm{~h}$ before spin coating. We obtained satisfactory films from the mixtures of $\alpha$ M-CMS-PSt and $\alpha$ M-CMS-PMSt, but no uniform film from the CMS-PMSt system for any mixture composition. In the CMS-PSt mixture, only the PSt rich composition gave good films, while in the PCSt-PSt system, the films were transparent, but not uniform in thickness, this being particularly so for the PSt- rich film.

The resist films were exposed to a $20 \mathrm{kV}$ electron beam using an electron beam exposure system ELS-5000 (Elionix). Exposure experiments were carried out only for transparent and uniform films. For the sensitivity measurements, rectangles $120 \mu \mathrm{m}$ long and $30 \mu \mathrm{m}$ wide were exposed for various times at a constant beam current. The initial film thickness was $0.8 \mu \mathrm{m}$, and the thicknesses before and after development were measured with a Talystep (Rank-Taylor-Hobson).

\section{RESULTS AND DISCUSSION}

Sensitivity curves of $\alpha$ M-CMS-PSt mixtures are shown in Figure 2, where the molecular weights of both polymers are about 40,000 . Surprisingly, despite the fact that the sensitivity of PSt was 30 times lower than that of $\alpha \mathrm{M}-\mathrm{CMS}$, no significant change occurred in the sensitivity curves on adding a substantial amount of PSt. The results are summarized in Figure 3, where three dosages, gel point dose " $D_{0}$," doses leaving half and $80 \%$ of the initial thickness " $D_{0.5}$ " and " $D_{0.8}$," are plotted against $\alpha$ M-CMS or PSt content. These dosages brought no significant change in the PSt 


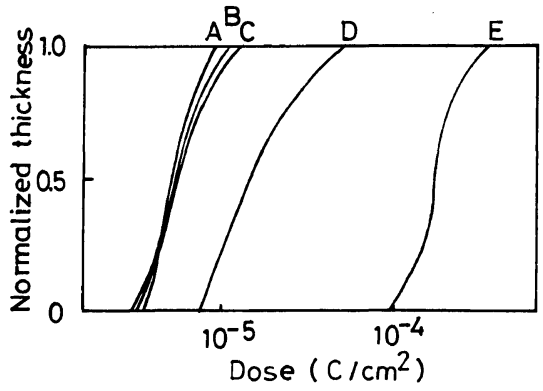

Figure 2. Sensitivity curves of $\alpha \mathrm{M}-\mathrm{CMS}-\mathrm{PSt}$ mixtures: A, $8 / 2 ; \mathrm{B}, 10 / 0 ; \mathrm{C}, 9 / 1 ; \mathrm{D}, 2 / 8 ; \mathrm{E}, 0 / 10$. The molecular weights of both polymers are about 40,000 . The initial resist thickness is $0.8 \mu \mathrm{m}$.

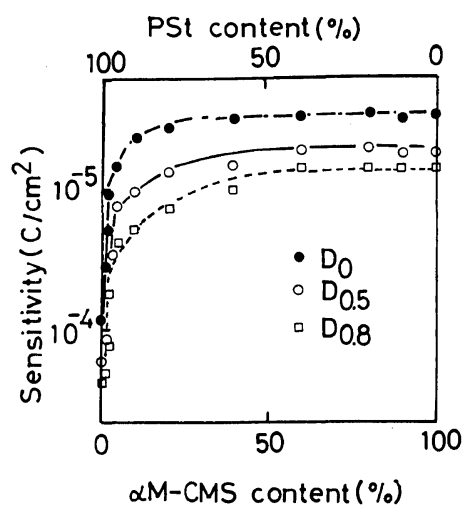

Figure 3. Variations in the three dosages, $D_{0}(\mathcal{O}), D_{0.5}$ ( $\bigcirc$ ), and $D_{0.8}(\square)$, as a function of $\alpha \mathrm{M}$-CMS content for $\alpha \mathrm{M}$-CMS-PSt mixtures. $D_{0}, D_{0.5}$, and $D_{0.8}$ indicate gel point dose doses, leaving half and $80 \%$ of the initial thickness respectively.

content of $0-50 \%$. That is, the contrast and sensitivity were kept constant up to a $50 \%$ PSt content. This clearly indicates that PSt crosslinks with $\alpha \mathrm{M}-\mathrm{CMS}$ with high efficiency. When the PSt content was larger than $60 \%$, the sensitivity of the mixture gradually decreased, and a sharp drop in sensitivity was occurred at a PSt content larger than $90 \%$. This tendency was at first observed in $D_{0.8}$, and then in $D_{0.5} . D_{0}$ still remained constant up to $90 \%$ of PSt content. These results mean that the contrast is gradually decreased with PSt content. It is significant that the curve of $D_{0.8}$ is almost identical with the dependence of the crosslinking reactivity

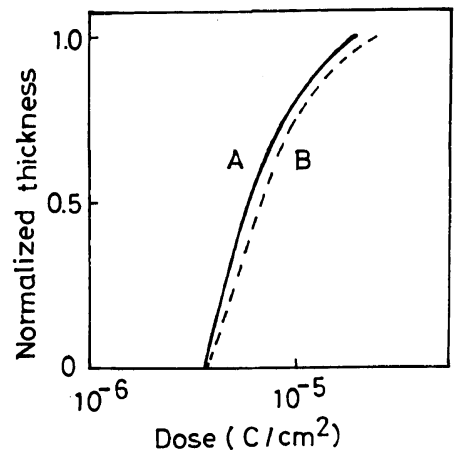

Figure 4. Sensitivity curves of the $4 / 6$ mixtures of $\alpha \mathrm{M}$ CMS and PSt. $M_{w}$ s of PSt are 100,000 (A) and 10,000 (B). $M_{w}$ of $\alpha \mathrm{M}-\mathrm{CMS}$ is 40,000 .

on the chloromethylation ratio in CMS and $\alpha \mathrm{M}-\mathrm{CMS}$. That is, the mixing of $\alpha \mathrm{M}-\mathrm{CMS}$ with PSt gives the same effect as the chloromethylation of PSt.

Figure 4 shows the sensitivity curves for the $4 / 6$ mixtures of $\alpha \mathrm{M}-\mathrm{CMS}\left(M_{w}=40,000\right)$ and PSt with $M_{w}$ of 10,000 and 100,000 . A large difference in $M_{w}$ of PSt did not affect the sensitivity of the mixture. This suggests that PSt actually crosslinks with $\alpha \mathrm{M}-\mathrm{CMS}$ but makes little contribution to the sensitivity. Since the sensitivity of crosslink-type negative electron resists is proportional to the $M_{w}$ of polymer, the crosslinking between the two PSt chains is negligible and the crosslinking between PSt and $\alpha \mathrm{M}-\mathrm{CMS}$ is as effective as that between the two $\alpha \mathrm{M}$-CMS chains. Such an effective reaction may be due to $\alpha$-hydrogen of PSt.

For confirmation of this, the sensitivity of the $\alpha$ M-CMS-PMSt system was measured. The $M_{w}$ of both polymers were 40,000. Despite their excellent film quality suggesting uniform mixing of the polymers, the developed pattern had numerous holes at any resist composition as shown in Figure 5. The size of the holes became larger with an increase in PMSt content possibly because the PMSt component is washed away from the resist films during development. An excess dosage ten times higher than $D_{0.8}$ of $\alpha \mathrm{M}-\mathrm{CMS}$ did not change this 


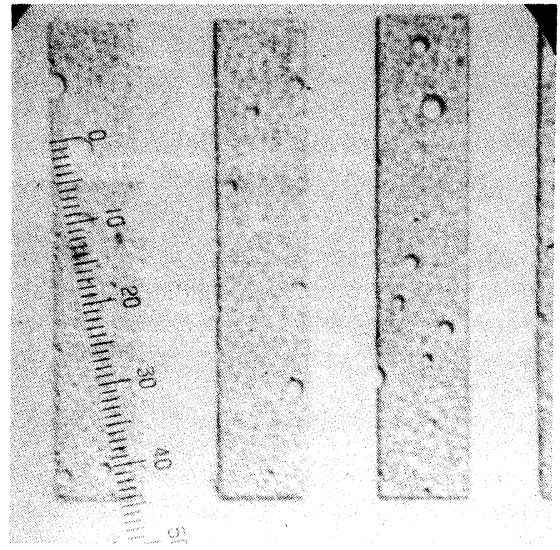

Figure 5. Photomicrograph of developed resist pattern for mixture of $\alpha \mathrm{M}-\mathrm{CMS}$ and PMSt (8/2) leaving about $100 \%$ of the initial thickness. $M_{w}$ s of both polymers are 40,000 . The holes in the rectangular pattern $120 \mu \mathrm{m}$ long and $30 \mu \mathrm{m}$ wide generated as a result of the PMSt component's being washed away from resist films with development.

situation. However, $D_{0}$ remained constant at almost all mixture compositions. These results clearly indicate that $\alpha \mathrm{M}-\mathrm{CMS}$ is virtually incapable of crosslinking with PMSt because of the lack of $\alpha$-hydrogen, and PMSt acts as a positive resist even in the mixture. Insoluble gel formed only by crosslinking of $\alpha \mathrm{M}-\mathrm{CMS}$ chains, and this way may explain the constant value of $D_{0}$. However, it is still not clear whether or not chlorine radicals formed by the dissociation of chloromethyl group in $\alpha \mathrm{M}$ CMS subtract the other hydrogen atoms from the PMSt structure. In conclusion, the methyl substitution of the $\alpha$-position greatly decreases the crosslinking reactivity of PSt.

Figure 6 shows the sensitivity change in CMS-PSt mixtures. Although we could not obtain sufficient data, the CMS-PSt system seems to give the same result as $\alpha \mathrm{M}$-CMS-PSt system. In Figure 7, the sensitivity curve for PCSt-PSt (6/4) mixture film is compared with that of PCSt. In PCSt, no sensitivity decrease occurs by the addition of $40 \%$ PSt, suggesting that the $\alpha$-hydrogen subtraction also occurs at a significant yield in PCSt.

As described above, in halogenated PSt, the

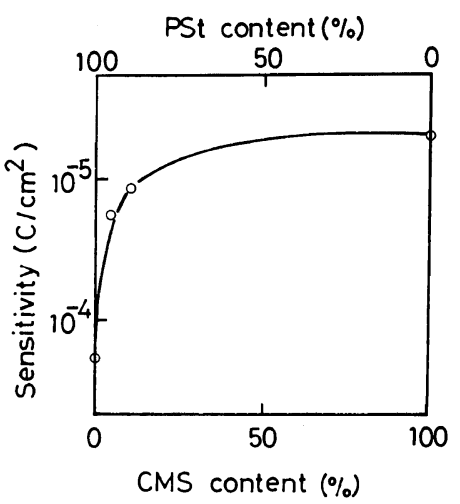

Figure 6. Variations in the dose $D_{0}$ of the mixture of CMS and PSt as a function of CMS content.

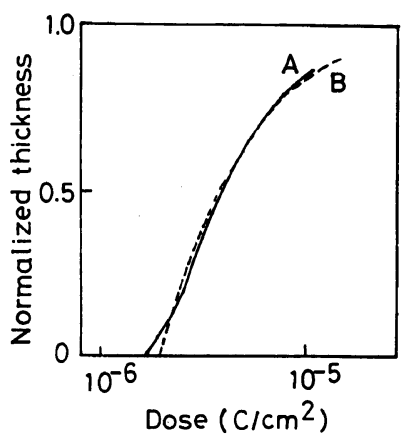

Figure 7. Sensitivity curves of PCSt (A) and PCSt-PSt (6/4) mixture films (B).

$\alpha$-hydrogen is easily subtracted by reactive intermediates, probably halogen radicals, and the benzyl-type radicals formed undergo the crosslinking reaction.

Acknowledgement. The authors thank Mr. K. Murase for his valuable comments and Miss K. Iimura for measuring the sensitivity curves.

\section{REFERENCES}

1. J. H. Lai and L. T. Shepherd, J. Electrochem. Soc., 126, 696 (1979).

2. E. D. Feit and L. E. Stillwagon, Polym. Eng. Sci., 17, 1058 (1980).

3. S. Imamura, J. Electrochem. Soc., 126, 1628 (1979).

4. H. Shiraishi, Y. Taniguchi, S. Horigome, and S. Nonogaki, Polym. Eng. Sci., 20, 1054 (1980).

5. K. Sukegawa and S. Sugawara, Jpn. J. Appl. Phys., 20, 1583 (1981). 